\title{
Effect of Prior History of Dementia and a Diagnosis of COVID-19 on Delirium in the ICU
}

\author{
Amira Kupty ${ }^{1}$, Fen-Lei Chang, MD, PhD² \\ ${ }^{1,2}$ Indiana University School of Medicine; ${ }^{2}$ Parkview Health Neuroscience
}

Background: Delirium occurred in nearly two out of every three patients in the ICU from prior studies. It is associated with poor health outcomes, including long-term cognitive and motor decline, increased risk of dementia, and increased mortality. In the context of COVID-19, patients are more likely to develop delirium due to the severity of the illness involving multiple organ systems. Our study evaluates factors contributing to the occurrence of delirium in the ICU for patients with or without a history of dementia and with or without a recent diagnosis of COVID-19.

Methods: We performed a retrospective chart review of ICU patients in Fort Wayne, IN from January through the end of June of 2020. Patients were grouped by whether they developed delirium or not and whether they had pre-existing dementia. We recorded age, sex, race, BMI, level of education, high risk medications, comorbidities, enteric/NPO feeding, mechanical ventilation, mobility, and lab results including CBC and BMP at the onset of delirium. Additionally, we included severity of delirium, length of stay, functional status at time of discharge, and COVID-19 status.

Results: We were unable to collect data at the time. However, we hypothesized that the incidence and severity of ICU delirium is associated with the severity of dementia and COVID status, among other factors.

Potential Impact: This study can shed light on the contribution of dementia history to ICU delirium and potential factors contributing to delirium incidence and severity. The COVID pandemic offers opportunities to differentiate effects of neurological (dementia) versus nonneurological factors (respiratory, renal, cardiovascular, and metabolic) on ICU delirium. 